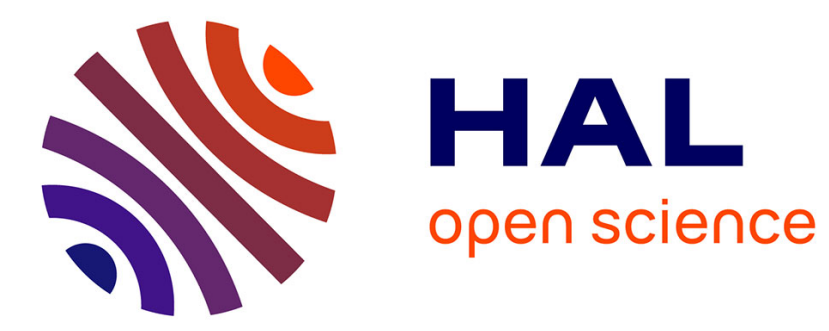

\title{
Static output feedback sliding mode control design via an artificial stabilizing delay
}

Alexandre Seuret, Chris Edwards, Sarah K. Spurgeon, Emilia Fridman

\section{To cite this version:}

Alexandre Seuret, Chris Edwards, Sarah K. Spurgeon, Emilia Fridman. Static output feedback sliding mode control design via an artificial stabilizing delay. IEEE Transactions on Automatic Control, 2009, 54 (2), pp.256 - 265. 10.1109/TAC.2008.2010889 . hal-00385893

\section{HAL Id: hal-00385893 https://hal.science/hal-00385893}

Submitted on 20 May 2009

HAL is a multi-disciplinary open access archive for the deposit and dissemination of scientific research documents, whether they are published or not. The documents may come from teaching and research institutions in France or abroad, or from public or private research centers.
L'archive ouverte pluridisciplinaire HAL, est destinée au dépôt et à la diffusion de documents scientifiques de niveau recherche, publiés ou non, émanant des établissements d'enseignement et de recherche français ou étrangers, des laboratoires publics ou privés. 


\title{
Static output feedback sliding mode control design via an artificial stabilizing delay
}

\author{
Alexandre Seuret, Christopher Edwards, Sarah K. Spurgeon and Emilia Fridman
}

\begin{abstract}
It is well known that for linear, uncertain systems, a static output feedback sliding mode controller can only be determined if a particular triple associated with the reduced order dynamics in the sliding mode is stabilisable. This paper shows that the static output feedback sliding mode control design problem can be solved for a broader class of systems if a known delay term is deliberately introduced into the switching function. Effectively the reduced order sliding mode dynamics are stabilized by the introduction of this artificial delay.
\end{abstract}

Index Terms-Sliding mode control, output feedback, time delay systems, exponential stability, discretized Lyapunov-Krasovskii functionals, stabilizing delay.

\section{INTRODUCTION}

In many practical situations, all the states are not available to the controller. In some circumstances it is impossible or prohibitively expensive to measure all of the process variables. With this in mind, many authors have developed methods to control systems only using output feedback, of which one approach is the output feedback sliding mode control paradigm [5].

The idea developed in this paper is to broaden the class of systems for which a static output feedback based sliding mode controller can be developed based on a recent result from time delay systems. In

The work by A. Seuret was partially supported by European Commission through the HYCON Network of Excellence, the Swedish Foundation for Strategic Research and by the Swedish Research Council, Automatic Control Department, KTH,SE-10044, Stockholm Sweden.e-mail: seuretakth. se.

C. Edwards, S.K. Spurgeon are with the Control and Instrumentation Research Group, Department of Engineering, University of Leicester, University Road, Leicester, LE1 7RH, UK. e-mail: ce14, eondle.ac.uk.

E. Fridman is with the Department of Electrical EngineeringSystems, Tel-Aviv University, Tel-Aviv 69978, Israel. emiliadeng.tau.ac.il:

Alexandre Seuret was supported by an EPSRC Platform Grant reference EP/D029937/1 entitled 'Control of Complex Systems'. S.K.Spurgeon, C.Edwards and E.Fridman gratefully acknowledge support from EPSRC Grant Reference entitled 'Robust Output Feedback Sliding Mode Control for Time-delay systems'.
[7], [11], the authors show that for some systems, the presence of delay can have a stabilizing effect. This affords the possibility of taking a system which is not stabilizable by static output feedback without delay and finding a constant delay $\tau$ strictly greater than 0 such that the system is stable. In this case, a stabilizing delay is introduced into the dynamics to effect output feedback stability.

This design concept is not new. Several authors have considered this possibility. For example in [15], [17], [18] it has been shown that introducing a delay in an output feedback controller can stabilize a system which cannot be stabilized without delay. This property has already been noted in the production of proteins in a cell [13]. When researchers try to model this production without delay, the solutions oscillate and do not correspond to the known physical behaviour. By introducing a delay corresponding to the intracellular transport by convection, the solutions correspond more closely to the known behavior.

The novelty in this paper is in overcoming the output feedback stabilizability assumption [2] in the design of sliding mode controllers by static output feedback. The authors propose a new switching function which contains an additional term which is linear in the delayed output. This is shown to be constructive in stabilizing the reduced order sliding mode dynamics. It is then shown that a sliding motion can be reached in finite time.

The article is organized as follows. The second section presents the problem formulation. Section three formulates the definition of a new sliding function which contains an artificial delay. In section four, the problem of exponential stability of the reduced order sliding motion with constant delay using discretized Lyapunov-Krasovskii functionals is solved. Section five deals with the exponential stabilization of non-delayed systems by a sliding mode controller including delay. In the last section, a numerical example demonstrates the design of the 
gains and the effect of the choice of the delay in the sliding mode controller.

Throughout the article, the notation $P>0$ for $P \in \mathbb{R}^{n \times n}$ means that $P$ is a symmetric and positive definite matrix. $\left[A_{1}\left|A_{2}\right| \ldots \mid A_{n}\right]$ is the concatenated matrix formed from the matrices $A_{i}$. The symbol $I_{n}$ represents the $n \times n$ identity matrix. The notations |.| and \|. || refer to the Euclidean vector norm and its induced matrix norm, respectively. For

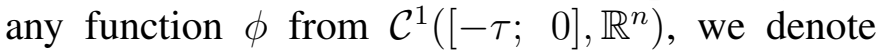
$|\phi|_{\tau}=\sup _{s \in[-\tau, 0]}(|\phi(s)|)$.

\section{PRELIMINARIES AND PROBLEM FORMULATION}

Consider the linear uncertain system without delay

$$
\begin{aligned}
& \dot{x}(t)=A x(t)+B(u(t)+\psi(y(t))) \\
& y(t)=C x(t)
\end{aligned}
$$

where $x(t) \in \mathbb{R}^{n}, u(t) \in \mathbb{R}^{m}$ and $y(t) \in \mathbb{R}^{p}$ with $m<p<n$, corresponds to the state, control and output variables respectively. The function $\psi \in \mathbb{R}^{m}$ represents the matched disturbances and is assumed to satisfy:

$$
\|\psi(t)\| \leq \Psi_{2}(y(t))
$$

where $\Psi_{2}$ is a known function.

The matrices $A \in \mathbb{R}^{n \times n}, B \in \mathbb{R}^{n \times m}, C \in \mathbb{R}^{p \times n}$ are assumed to be known. It is also assumed that the pair $(A, B)$ is controllable and the input and the output matrices $B$ and $C$ are full rank. In addition, it is assumed $\operatorname{rank}(C B)=m$. Then from [2], [4], there exists a change of variables such that the system has the following representation:

$$
\begin{aligned}
& \dot{x}(t)=\left[\begin{array}{cc}
A_{11} & A_{12} \\
A_{21} & A_{22}
\end{array}\right] x(t)+\left[\begin{array}{c}
0 \\
B_{2}
\end{array}\right](u(t)+\psi(y(t))) \\
& y(t)=\left[\begin{array}{ll}
0 & T
\end{array}\right] x(t)
\end{aligned}
$$

where $A_{11} \in \mathbb{R}^{(n-m) \times(n-m)}, B_{2} \in \mathbb{R}^{m \times m}$ is nonsingular and $T \in \mathbb{R}^{p \times p}$ is an orthogonal matrix. In [2] a sliding surface

$$
\mathbb{S}=\left\{x \in \mathbb{R}^{n}: F C x(t)=0\right\}
$$

is proposed, where $F=F_{2}\left[\begin{array}{ll}K & I_{m}\end{array}\right] T^{T}, K \in$ $\mathbb{R}^{m \times(p-m)}$ and $F_{2} \in \mathbb{R}^{m \times m}$ is a nonsingular matrix. The sliding motion is governed by the choice of $K$. If a further coordinate change is introduced based on the nonsingular transformation $z=\hat{T} x$ with $\hat{T}$ defined by:

$$
\hat{T}=\left[\begin{array}{cc}
I_{n-m} & 0 \\
K C_{1} & I_{m}
\end{array}\right]
$$

where $C_{1}=\left[\begin{array}{ll}0_{(p-m) \times(n-p)} & I_{(p-m)}\end{array}\right]$, then, as argued in [2], the dynamics of the reduced order sliding motion is governed by

$$
\dot{x}_{1}=\left(A_{11}-A_{12} K C_{1}\right) x_{1}(t)
$$

The fictitious system $\left(A_{11}, A_{12}, C_{1}\right)$ is assumed to be output stabilizable i.e., there exist a matrix $K$ such that the matrix $A_{11}-A_{12} K C_{1}$ is Hurwitz. It is shown in [2] that a necessary condition for $\left(A_{11}, A_{12}, C_{1}\right)$ to be stabilizable is that the invariant zeros of $(A, B, C)$ lie in the open left half-plane. However the design of an output feedback gain $K$ such that the matrix $A_{11}+A_{12} K C_{1}$ is Hurwitz is not always straightforward and may be impossible.Consider for instance the system (6) with

$A_{11}=\left[\begin{array}{cc}0 & -2 \\ 1 & 0.1\end{array}\right], A_{12}=\left[\begin{array}{c}-1 \\ 0\end{array}\right], C_{1}=\left[\begin{array}{ll}0 & 1\end{array}\right]$ which is from [1], [2]. In this case, the output feedback stabilization problem becomes the problem of finding a scalar $k$ such that the matrix $\left[\begin{array}{cc}0 & -2-k \\ 1 & 0.1\end{array}\right]$ has strictly negative eigenvalues, which is clearly not possible. In this situation, some authors [1], [3], [5] have employed a compensator in order to stabilize the system. However, these methods increase the order of the controller and have an associated computational overhead both in terms of design and implementation. The proposed method seeks to introduce an artificial delay in the system such that the system can be stabilized by static output feedback without the need to introduce a compensator.

\section{DESIGN OF A NEW SLIDING MODE SURFACE}

In this section, the design of a new type of sliding surface will be discussed. The objective is to define a sliding surface of the form of (4) but which introduces a delay in the reduced order dynamics. Consider

$$
\mathbb{S}^{\prime}=\left\{x \in \mathbb{R}^{n}: F C x(t)+F_{\tau} C x(t-\tau)=0\right\}
$$

where as before the matrix $F=F_{2}\left[\begin{array}{ll}K & I_{m}\end{array}\right] T^{T}$ and where $F_{\tau}=F_{2}\left[K_{\tau} 0_{m}\right] T^{T}, K_{\tau} \in \mathbb{R}^{m \times(p-m)}$. Here, 
without loss of generality, the matrices $F_{2}$ and $T$ are chosen as $I_{m}$. In (7), $\tau$ is an artificial, fixed and known delay which has to be chosen to stabilize the reduced order dynamics in the sliding mode and represents a design parameter. The existence of such a delay and constructive methods to choose it will be discussed in a latter section. Instead of (5), consider the coordinate change $x \mapsto T_{\tau} x$ :

$$
\begin{aligned}
& \tilde{x}_{1}(t)=x_{1}(t) \\
& \tilde{x}_{2}(t)=x_{2}(t)+K C_{1} x_{1}(t)+K_{\tau} C_{1} x_{1}(t-\tau)
\end{aligned}
$$

By construction the switching function associated with $\mathbb{S}^{\prime}$ is $s(t)=\tilde{x}_{2}(t)$. This leads to:

$$
\begin{aligned}
& \dot{\tilde{x}}_{1}(t)=\left(A_{11}-A_{12} K C_{1}\right) \tilde{x}_{1}(t) \\
& \quad-A_{12} K_{\tau} C_{1} \tilde{x}_{1}(t-\tau)+A_{12} \tilde{x}_{2}(t) \\
& \quad \dot{\tilde{x}}_{2}(t)=\left(A_{21}+K C_{1} A_{11}\right) \tilde{x}_{1}(t) \\
& \quad+K_{\tau} C_{1} A_{11} \tilde{x}_{1}(t-\tau)+\left(A_{22}+K C_{1} A_{12}\right) \tilde{x}_{2}(t) \\
& \quad+K_{\tau} C_{1} A_{12} \tilde{x}_{2}(t-\tau)+B_{2}(u(t)+\psi(t)) \\
& \quad-\left(A_{22}+K C_{1} A_{12}\right) K C_{1} \tilde{x}_{1}(t)-\left(K C_{1} A_{12} K_{\tau}\right. \\
& \left.\quad+A_{22} K_{\tau}+K_{\tau} C_{1} A_{12} K\right) C_{1} \tilde{x}_{1}(t-\tau) \\
& \quad-K_{\tau} C_{1} A_{12} K_{\tau} C_{1} \tilde{x}_{1}(t-2 \tau)
\end{aligned}
$$

Remark 1: It is important to note that the system (8) is a particular delay system. Since the delay is artificially introduced in the sliding manifold, the delay $\tau$ is known and can be chosen to improve the stability of the closed-loop system.

Remark 2: The sliding mode dynamics are given by equation (8) with $\tilde{x}_{2}(t)=0$. This is a retarded system, where the delay is known and can be selected to stabilise, or enhance the stability of, the reduced order sliding motion.

Remark 3: Note that the range space dynamics given in (9) contain several delayed terms and two different delays, $\tau$ and $2 \tau$. However $\tau$ is a design parameter in the particular formulation presented here, and thus $\tau$ is perfectly known to the controller.

The last two lines of equation (9) only depend on the known output information, $\tilde{x}_{2}$ and $C_{1} \tilde{x}_{1}$, where $T^{T} y=\left[C_{1} \tilde{x}_{1}, \tilde{x}_{2}\right]$, and thus the following output feedback control law can be defined:

$$
\begin{aligned}
u(t) & =-\left(B_{2}\right)^{-1}\left\{\left(A_{22}+K C_{1} A_{12}\right) \tilde{x}_{2}(t)\right. \\
& +K_{\tau} C_{1} A_{12} \tilde{x}_{2}(t-\tau) \\
& -\left(A_{22}+K C_{1} A_{12}\right) K\left(C_{1} \tilde{x}_{1}(t)\right) \\
& -K_{\tau} C_{1} A_{12} K_{\tau}\left(C_{1} \tilde{x}_{1}(t-2 \tau)\right) \\
& -G_{l} \tilde{x}_{2}(t)+\nu(t)-\left(K C_{1} A_{12} K_{\tau}\right. \\
& \left.\left.+A_{22} K_{\tau}+K_{\tau} C_{1} A_{12} K\right)\left(C_{1} \tilde{x}_{1}(t-\tau)\right)\right\}
\end{aligned}
$$

where $\tilde{x}_{i}(t)=0, t<0, i=1,2$ and $G_{l}$ is a Hurwitz matrix. The term $\nu$ is the discontinuous injection defined by

$$
\nu(t)= \begin{cases}\rho(t, y) \frac{Q_{2} \tilde{x}_{2}(t)}{\left\|Q_{2} \tilde{x}_{2}(t)\right\|} & \text { if } \tilde{x}_{2}(t) \neq 0 \\ 0 & \text { otherwise }\end{cases}
$$

where $Q_{2}$ is a symmetric positive definite matrix in $\mathbb{R}^{m \times m}$ and

$$
\rho=\left\|B_{2}\right\| \Psi_{2}(y(t))+\delta
$$

where $\delta$ is a positive scalar gain. The closed loop system satisfies the following equations

$$
\begin{aligned}
\dot{\tilde{x}}_{1}(t) & =\left(A_{11}-A_{12} K C_{1}\right) \tilde{x}_{1}(t) \\
& -A_{12} K_{\tau} C_{1} \tilde{x}_{1}(t-\tau)+A_{12} \tilde{x}_{2}(t) \\
\dot{\tilde{x}}_{2}(t) & =\left(A_{21}+K C_{1} A_{11}\right) \tilde{x}_{1}(t)+G_{l} \tilde{x}_{2}(t) \\
& +K_{\tau} C_{1} A_{11} \tilde{x}_{1}(t-\tau)-\nu(t)+B_{2} \psi(y(t))
\end{aligned}
$$

Remark 4: Note that the control law (10) does not have a heavy computational overhead.

\section{EXPONENTIAL STABILITY OF THE CLOSED LOOP SYSTEM}

\section{A. Exponential stability of the reduced order system}

Consider the linear system with constant delay:

$$
\dot{\tilde{x}}_{1}(t)=A_{0} \tilde{x}_{1}(t)+A_{1} \tilde{x}_{1}(t-\tau)
$$

where $\tilde{x}_{1} \in \mathbb{R}^{(n-m)}$ is the state and where $A_{0}=$ $A_{11}-A_{12} K C_{1}$ and $A_{1}=-A_{12} K_{\tau} C_{1}$ are constant matrices with appropriate dimensions. System (14) represents the dynamics of the reduced order system (8) when $\tilde{x}_{2}(t)=0$. Therefore, the sliding surface (7) underpins the stabilization of the sliding mode dynamics by using the delayed term $A_{12} K_{\tau} C_{1} x_{1}(t-$ $\tau)$.

System (14) is said to be exponentially stable [14], [16] with a decay rate $\alpha>0$ and an exponential gain $\beta \geq 1$ if the following exponential bound holds:

$$
\left|\tilde{x}_{1}\left(t ; t_{0}, \phi\right)\right|<\beta|\phi|_{\tau_{2}} e^{-\alpha\left(t-t_{0}\right)},
$$

where $\tilde{x}_{1}\left(t ; t_{0}, \phi\right)$ is the solution of (14), starting at time $t_{0}$ from the initial function $\phi \in \mathcal{C}^{1}$. Note that both $\alpha$ and $\beta$ must be independent of $\phi$.

Consider the change of variable $x_{\alpha}(t)=e^{\alpha t} \tilde{x}_{1}(t)$ as in [19], [21]. Effectively, asymptotic convergence of the $x_{\alpha}$ states implies exponential convergence of 
$\tilde{x}_{1}$ at a prescribed rate. Then it is easy to see that in the case of constant delay, equation (14) becomes

$$
\dot{x}_{\alpha}(t)=\left(A_{0}+\alpha I_{n}\right) x_{\alpha}(t)+e^{\alpha \tau} A_{1} x_{\alpha}(t-\tau)
$$

Consider the following theorem based on the $N$ discretized Lyapunov-Krasovskii functional proposed in [11].

Theorem 1: System (14) is exponentially stable with the decay rate $\alpha$ if there exist $(n-m) \times(n-m)$ matrices $P_{1}>0, P_{2}, P_{3}, S_{p}=S_{p}^{T}, Q_{p}, R_{p q}=R_{q p}^{T}$, $p, q=0, \ldots, N$, which satisfy the LMI conditions (17) and (18) with $h=\tau / N$

$$
\Pi_{\alpha}=\left[\begin{array}{ccc}
\Xi_{\alpha} & D^{s} & D^{a} \\
* & -R_{d}-S_{d} & 0 \\
* & * & -3 S_{d}
\end{array}\right]<0
$$

and

$$
\left[\begin{array}{cc}
P_{1} & \tilde{Q} \\
* & \tilde{R}+\tilde{S}
\end{array}\right]>0
$$

where the matrix $\Xi_{\alpha}$ is given by

$$
\left[\begin{array}{cc}
\Psi_{\alpha} & P^{T}\left[\begin{array}{c}
0_{n} \\
e^{\alpha \tau} A_{1}
\end{array}\right]-\left[\begin{array}{c}
Q_{N} \\
0_{n}
\end{array}\right] \\
* & -S_{N}
\end{array}\right]
$$

and where $\Psi_{\alpha}$ is given by

$$
\begin{aligned}
& P^{T}\left[\begin{array}{cc}
0_{n} & I_{n} \\
A_{0}+\alpha I_{n} & -I_{n}
\end{array}\right]+\left[\begin{array}{cc}
0_{n} & I_{n} \\
A_{0}+\alpha I_{n} & -I_{n}
\end{array}\right]^{T} P \\
& +\left[\begin{array}{cc}
Q_{0}+Q_{0}^{T}+S_{0} & 0_{n} \\
0_{n} & 0_{n}
\end{array}\right]
\end{aligned}
$$

where

$$
\begin{aligned}
& \tilde{Q}=\left[\begin{array}{llll}
Q_{0} & Q_{1} & \ldots & Q_{N}
\end{array}\right], \\
& \tilde{S}=\operatorname{diag}\left\{1 / h S_{0}, 1 / h S_{1}, \ldots, 1 / h S_{N}\right\} \text {, } \\
& \tilde{R}=\left[\begin{array}{cccc}
R_{00} & R_{01} & \ldots & R_{0 N} \\
R_{01} & R_{11} & \ldots & R_{1 N} \\
\vdots & \vdots & \ddots & \vdots \\
R_{N 0} & R_{N 1} & \ldots & R_{N N}
\end{array}\right] . \\
& P=\left[\begin{array}{ll}
P_{1} & 0_{n} \\
P_{2} & P_{3}
\end{array}\right]
\end{aligned}
$$

and where for $i, j=1, . ., N$

$$
\begin{aligned}
& S_{d}=\operatorname{diag}\left\{S_{0}-S_{1}, S_{1}-S_{2}, \ldots, S_{N-1}-S_{N}\right\}, \\
& R_{d i j}=h\left(R_{(i-1)(j-1)}-R_{i j}\right), \\
& R_{d}=\left[\begin{array}{cccc}
R_{d 11} & R_{d 12} & \ldots & R_{d 1 N} \\
R_{d 21} & R_{d 22} & \ldots & R_{d 2 N} \\
\vdots & \vdots & \ddots & \vdots \\
R_{d N 1} & R_{d N 2} & \ldots & R_{d N N}
\end{array}\right] \text {, } \\
& D^{s}=\left[\begin{array}{llll}
D_{1}^{s} & D_{2}^{s} & \ldots & D_{N}^{s}
\end{array}\right], \\
& D_{i}^{s}=\left[\begin{array}{c}
\left(R_{0(i-1)}+R_{0 i}\right)-\left(Q_{i-1}-Q_{i}\right) \\
h / 2\left(Q_{i-1}+Q_{i}\right) \\
-h / 2\left(R_{N(i-1)}+R_{N i}\right)
\end{array}\right] \text {, } \\
& D^{a}=\left[\begin{array}{llll}
D_{1}^{a} & D_{2}^{a} & \ldots & D_{N}^{a}
\end{array}\right], \\
& D_{i}^{a}=\left[\begin{array}{c}
-h / 2\left(R_{0(i-1)}-R_{0 i}\right) \\
-h / 2\left(Q_{i-1}-Q_{i}\right) \\
h / 2\left(R_{N(i-1)}-R_{N i}\right)
\end{array}\right],
\end{aligned}
$$

Proof: Consider the following LyapunovKrasovskii functional:

$$
\begin{aligned}
& V_{1 \alpha}(t)=x_{\alpha}^{T}(t) P_{1} x_{\alpha}(t) \\
& \quad+2 x_{\alpha}^{T}(t) \int_{-\tau}^{0} Q(\xi) x_{\alpha}(t+\xi) d \xi \\
& \quad+\int_{-\tau}^{0} x_{\alpha}^{T}(t+\xi) S(\xi) x_{\alpha}(t+\xi) d \xi \\
& \quad+\int_{-\tau}^{0} \int_{-\tau}^{0} x_{\alpha}^{T}(t+s) R(s, \xi) d s x_{\alpha}(t+\xi) d \xi
\end{aligned}
$$

where $P_{1}>0, Q(\xi) \in \mathbb{R}^{(n-m) \times(n-m)}, R(s, \xi)=$ $R^{T}(\xi, s) \in \mathbb{R}^{(n-m) \times(n-m)}, S(\xi) \in \mathbb{R}^{(n-m) \times(n-m)}$, and $Q, R, S$ are continuous matrix functions. From [12] (p. 185) $V_{1 \alpha}$ is positive definite if the LMI (18) holds. Then the proof follows along the lines of [7] using a descriptor representation [9] and Gu-discretization [11]. It follows that $x_{\alpha}$ converges asymptotically to the solution $x_{\alpha}=0$ and consequently, the variable $x$ converges exponentially to the solution $x=0$ with the decay rate $\alpha$. See the Appendix for more details.

Remark 5: Note that Theorem 1 is an extension of Theorem 2.1 from [7] to the exponential stability case. However the exponential stability considerations allow the performance and the convergence of the solutions to be characterized, which will be efficient for the design of the output feedback controller.

Remark 6: In the definition of the delayed sliding manifold (7), the delay is chosen to be constant. If for some reason the chosen delay needs to be time-varying, then a time-varying gain $1-\dot{\tau}(t)$ will appear in the control law and the change of variables ' $x \rightarrow x_{\alpha}$ ' will affect system (16) as the exponential gain will also be time-varying. However this situation can also be dealt with: see for example [19] or [20].

\section{B. Illustrative example}

Consider system (14) [8], [10] with

$$
A_{0}=\left[\begin{array}{cc}
0 & 1 \\
-2 & 0.1
\end{array}\right], \quad A_{1}=\left[\begin{array}{ll}
0 & 0 \\
1 & 0
\end{array}\right]
$$

As in [8], Theorem 1 cannot guarantee that this system is asymptotically stable, i.e. for $\alpha=0$, if the delay is less than $\tau_{\min }=0.11 \mathrm{~s}$. The relationship between the delay $\tau$ and the maximum admissible decay rate $\alpha$ is given in Figure 1. The maximal decay rate $\alpha$ results from the following optimization problem (see the Appendix, section B for more 
details):

$$
\alpha_{\max }=\max _{\tau \in[0,2]} \alpha
$$

such that (17) and (18) are satisfied

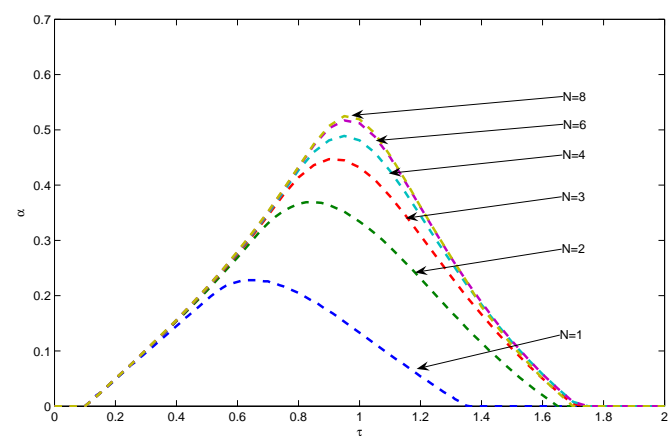

Fig. 1. Relation between $\tau$ and $\alpha$ with respect to $N$

Figure 1 shows that the conservatism of the condition from Theorem 1 reduces when the number of discretizations $N$ is increased. This is due to the fact that when $N$ increases, the degree of freedom to define the Lyapunov-Krasovskii functional also increases. Note that for all the discretizations, there exists a optimal delay which corresponds to the maximal decay rate. In a system where the delay can be chosen, as in system (13) presented in Section III, this form of graph can help to determine the optimal delay. Compared to the asymptotic result proposed in [8], Theorem 1 allows the existence of an optimal delay to be shown. This delay corresponds to the best performance in terms of stability.

Remark 7: Note that the 'optimal delay' is relative to the number $N$ of discretizations used in Theorem 1. In Figure 1 the optimal delay when $N=1$ is different from the one when $N=2$. In the sequel the statement 'optimal delay' will be used to express the delay which corresponds to the fastest decay rate $\alpha$ with respect to a certain level of discretization.

\section{Stabilization of the closed loop system}

This section focusses on the stability of the whole system (13). In particular, it needs to be established that $\tilde{x}_{2}=0$ in finite time, i.e. a sliding motion is achieved.

Theorem 2: System (13) is exponentially stable for given output feedback gains $K$ and $K_{\tau}$ with decay rate $\alpha$ if there exist $P_{1}>0, P_{2}, P_{3}, S_{p}=S_{p}^{T}$,
$Q_{p}, R_{p q}=R_{q p}^{T}, p, q=0, \ldots, N$ in $\mathbb{R}^{(n-m) \times(n-m)}$ and $Q_{2}>0 \in \mathbb{R}^{m \times m}$ which satisfy the LMI condition (21) and (18) with $h=\tau / N$

$$
\left.\left[\begin{array}{ccc} 
& & {\left[\begin{array}{c}
\left(A_{21}+K C_{1} A_{11}\right)^{T} Q_{2}+P_{1} A_{12} \\
0_{\alpha}(n-m) \times m \\
\\
\end{array}\right.} \\
e^{\alpha \tau}\left(K_{\tau} C_{1} A_{11}\right)^{T} Q_{2} \\
0_{N(n-m) \times m} \\
0_{N(n-m) \times m} \\
Q_{2} G_{l}+G_{l}^{T} Q_{2}+2 \alpha Q_{2}
\end{array}\right]\right]<0
$$

where the matrix $\Pi_{\alpha}$ is given by (19) and where $A_{0}=A_{11}-A_{12} K C_{1}$ and $A_{1}=-A_{12} K C_{1}$.

Proof: Consider new variables $\tilde{x}_{1 \alpha}(t)=$ $\tilde{x}_{1}(t) e^{\alpha t}$ and $\tilde{x}_{2 \alpha}(t)=\tilde{x}_{2}(t) e^{\alpha t}$. The new closedloop system satisfies the following equations:

$$
\begin{aligned}
& \dot{\tilde{x}}_{1 \alpha}(t)=\left(A_{11}-A_{12} K C_{1}+\alpha I_{n-m}\right) \tilde{x}_{1 \alpha}(t) \\
& \quad-e^{\alpha \tau} A_{12} K_{\tau} C_{1} \tilde{x}_{1 \alpha}(t-\tau)+A_{12} \tilde{x}_{2 \alpha}(t) \\
& \quad \dot{\tilde{x}}_{2 \alpha}(t)=\left(A_{21}+K C_{1} A_{11}\right) \tilde{x}_{1 \alpha}(t) \\
& \quad+e^{\alpha \tau} K_{\tau} C_{1} A_{11} \tilde{x}_{1 \alpha}(t-\tau) \\
& \quad+\left(G_{l}+\alpha I_{m}\right) \tilde{x}_{2 \alpha}(t)-e^{\alpha t}\left(\nu(t)-B_{2} \psi(y(t))\right)
\end{aligned}
$$

Consider the Lyapunov-Krasovskii functional

$$
V_{\alpha}(t)=V_{1 \alpha}(t)+V_{2 \alpha}(t)
$$

where $V_{1 \alpha}$ is defined in (20) and where

$$
V_{2 \alpha}(t)=x_{2 \alpha}^{T}(t) Q_{2} x_{2 \alpha}(t)
$$

From [7] and following the line of the proof proposed in the appendix, differentiating $V_{1 \alpha}$ along the trajectory of (22a) leads to the following inequality:

$$
\begin{aligned}
\dot{V}_{1 \alpha} \leq & \xi^{T}(t) \Xi_{\alpha} \xi(t)-\int_{0}^{1} \phi^{T}(\beta) S_{d} \phi(\beta) d \beta \\
& -\int_{0}^{1} \int_{0}^{1} \phi^{T}(\beta) R_{d} \phi(\gamma) d \beta d \gamma \\
& +2 \xi^{T}(t) \int_{0}^{1}\left[D^{s}+(1-2 \beta) D^{a}\right] \phi(\beta) d \beta \\
& +\tilde{x}_{1 \alpha}^{T}(t) P_{1} A_{12} \tilde{x}_{2 \alpha}(t)
\end{aligned}
$$

where $\Xi_{\alpha}$ is defined in (19) and the functions $\xi$ and $\phi$ are defined in the appendix.

Differentiating $V_{2 \alpha}$ along the trajectory of (22a) leads to:

$$
\begin{aligned}
\dot{V}_{2 \alpha} \leq & \tilde{x}_{2 \alpha}^{T}(t)\left(G_{l}^{T} Q_{2}+Q_{2} G_{l}+2 \alpha I_{m}\right) \tilde{x}_{2 \alpha}(t) \\
& +x_{2 \alpha}^{T}(t) Q_{2}\left[\left(A_{21}+K C_{1} A_{11}\right) \tilde{x}_{1 \alpha}(t)\right. \\
& +e^{\alpha \tau} K_{\tau} C_{1} A_{11} \tilde{x}_{1 \alpha}(t-\tau)-e^{\alpha t}(\nu(t) \\
& \left.\left.+B_{2} \psi(y(t))\right)\right]
\end{aligned}
$$

Then by combining (23) and (24) and by defining $\xi^{\prime}(t)=\operatorname{col}\left\{\tilde{x}_{1 \alpha}(t), \dot{\tilde{x}}_{1 \alpha}(t), \tilde{x}_{1 \alpha}(t-\tau), \tilde{x}_{2 \alpha}(t)\right\}$, the following inequality holds: 


$$
\begin{aligned}
\dot{V}_{\alpha} \leq & \xi^{T}(t) \Theta_{\alpha} \xi^{\prime}(t)-\int_{0}^{1} \phi^{T}(\beta) S_{d} \phi(\beta) d \beta \\
& -\int_{0}^{1} \int_{0}^{1} \phi^{T}(\beta) R_{d} \phi(\gamma) d \beta d \gamma \\
& +2 \xi^{T}(t) \int_{0}^{1}\left[D^{s}+(1-2 \beta) D^{a}\right] \phi(\beta) d \beta \\
& -x_{2 \alpha}^{T}(t) Q_{2} e^{\alpha t}\left(\nu(t)-B_{2} \psi(y(t))\right)
\end{aligned}
$$

where $\Theta_{\alpha}$ is given by:

$$
\left.\left[\begin{array}{cc}
\Xi_{\alpha} & {\left[\begin{array}{c}
\left(A_{21}+K C_{1} A_{11}\right)^{T} Q_{2}+P_{1} A_{12} \\
0_{(n-m) \times m} \\
*
\end{array}\right.} \\
e^{\alpha \tau}\left(K_{\tau} C_{1} A_{11}\right)^{T} Q_{2} \\
G_{l}^{T} Q_{2}+Q_{2} G_{l}
\end{array}\right]\right]
$$

From (12), note that $-x_{2 \alpha}^{T}(t) Q_{2} e^{\alpha t}(\nu(t)-$ $\left.B_{2} \psi(y(t))\right) \leq-\delta e^{\alpha t}\left\|Q_{2} y(t)\right\|$. The last term is thus negative. Applying Proposition 5.21 from [12] to (25) it can be concluded that $\dot{V}(t)<0$ if LMI (21) holds.

\section{Reachability of the sliding manifold in finite time}

Corollary 1: An ideal sliding motion takes place on the surface $s(t)=0$ in the domain $\Omega=$ $\left\{\left(\tilde{x}_{1}, \tilde{x}_{2}\right) \in[t-\tau, t] \mapsto \mathbb{R}^{n-m} \times \mathbb{R}^{m}:\left(\|\left(A_{21}+\right.\right.\right.$ $\left.\left.\left.K C_{1} A_{11}\right)\|+\| K_{\tau} C_{1} A_{11} \|\right)\left|\tilde{x}_{1}\right|_{\tau}<\delta-\eta\right\}$ where $\eta$ is a small scalar satisfying $0<\eta<\delta$.

Proof: Consider the following Lyapunov function $V_{s}(t)=\tilde{x}_{2}^{T}(t) Q_{2} \tilde{x}_{2}(t)$. By differentiating $V_{s}$ along the trajectories of (13b), it follows that:

$$
\begin{aligned}
\dot{V}_{s}(t) & =\tilde{x}_{2}^{T}(t)\left(Q_{2} G_{l}+G_{l}^{T} Q_{2}\right) \tilde{x}_{2}(t) \\
& +2 \tilde{x}_{2}^{T}(t) Q_{2}\left[\left(A_{21}-K C_{1} A_{11}\right) \tilde{x}_{1}(t)\right. \\
& \left.-K_{\tau} C_{1} A_{11} \tilde{x}_{1}(t-\tau)-\nu(t)+B_{2} \psi(y(t))\right]
\end{aligned}
$$

Since the matrix $G_{l}$ is Hurwitz, $Q_{2}$ can be chosen such that $Q_{2} G_{l}+G_{l}^{T} Q_{2}<0$. By taking an upper bound on the second and third term, the following inequality holds:

$$
\begin{aligned}
\dot{V}_{s}(t) & \leq 2\left\|\tilde{x}_{2}\right\|\left\|Q_{2}\right\|\left[\left(\left\|\left(A_{21}+K C_{1} A_{11}\right)\right\|\right.\right. \\
& \left.\left.+\left\|K_{\tau} C_{1} A_{11}\right\|\right)\left|\tilde{x}_{1}\right|_{\tau}+\left\|B_{2}\right\| \| \psi(y(t)) \mid\right] \\
& -\left\|\tilde{x}_{2}\right\|\left\|Q_{2}\right\| \rho(t, y)
\end{aligned}
$$

If the system satisfies the conditions from Theorem 2 , the state $\tilde{x}_{1}$ converges to the solution $\tilde{x}_{1}=0$ with an exponential decay rate. It follows that the domain $\Omega$ is reached in finite time. Since the gain $\rho$ of the sliding function is defined as $\rho(t, y)=\Psi_{2}(y(t))+\delta$, the following inequality holds:

$$
\dot{V}_{s}(t) \leq-\eta \sqrt{V_{s}}(t)
$$

This concludes the proof.
E. Comments on the design of the output feedback gain

As usual, the problem of designing the output feedback gain is not straightforward. Moreover the LMI (21) is not in an appropriate form for synthesis purposes because the gains $K$ and $K_{\tau}$ appear in different ways in $\Xi_{\alpha}$ than in $\left(K C_{1} A_{11}\right)^{T} Q_{2}$ and $\left(K_{\tau} C_{1} A_{11}\right)^{T} Q_{2}$. Congruence and other 'classical' LMI transformations will probably not facilitate constructive conditions. A constructive method at this time is to test the stability of the closed-loop system for a given set of values of $K$ and $K_{\tau}$ is discussed in the appendix.

\section{EXTENSION TO UNCERTAIN SYSTEMS}

Consider now the case when the system (3) is uncertain and time varying. Instead of the known matrices $A_{k l}$ for $k, l=1,2$, the following representation is introduced:

$$
\begin{aligned}
& A_{k l}^{t}=A_{k l}^{0}+\sum \underset{i=1}{M} \lambda_{i}(t) A_{k l}^{i}, \\
& B_{2}^{t}=B_{20}+\sum{ }_{i=1}^{M} \lambda_{i}(t) B_{2}^{i}
\end{aligned}
$$

where $A_{11}^{0} \in \mathbb{R}^{(n-m) \times(n-m)}$ and $B_{20} \in \mathbb{R}^{m \times m}$ is non singular. The other matrices in (26) are assumed to have appropriate dimensions. It is assumed that, for all $i \in\{1, . ., M\}$, the pair of matrices $\left(A_{k l}^{0}+\right.$ $\left.A_{k l}^{i}, B_{20}\right)$ is controllable. The scalar functions $\lambda_{i}$ are such that:

$$
\forall i=1, . ., M, \lambda_{i}(t) \in[0, \quad 1], \sum_{i=1}^{M} \lambda_{i}(t)=1
$$

As it is possible to remove some uncertainties, the system is rewritten as:

$$
\begin{aligned}
\dot{x}(t) & =\left[\begin{array}{cc}
A_{11}^{t} & A_{12}^{t} \\
A_{21}^{t} & A_{22}^{0}
\end{array}\right] x(t) \\
& +\left[\begin{array}{c}
0 \\
B_{20}
\end{array}\right]\left(u(t)+\psi_{0}(t, y, u)\right) \\
y(t) & =\left[\begin{array}{ll}
0 & T
\end{array}\right] x(t)
\end{aligned}
$$

where the matched uncertainties are represented by:

$$
\begin{aligned}
\psi_{0}(t, y, u)= & B_{20}^{-1}\left(\sum_{i=1}^{M} \lambda_{i}(t)\left(A_{22}^{i} x_{2}(t)+B_{2}^{i} u(t)\right)\right) \\
& +\psi(t, y)
\end{aligned}
$$


This leads to

$$
\begin{aligned}
\dot{\tilde{x}}_{1}(t) & =\left(A_{11}^{t}-A_{12}^{t} K C_{1}\right) \tilde{x}_{1}(t)+A_{12}^{t} \tilde{x}_{2} \\
& -A_{12}^{t} K_{\tau} C_{1} \tilde{x}_{1}(t-\tau) \\
\dot{\tilde{x}}_{2}(t) & =\left(A_{21}^{t}+K C_{1} A_{11}^{t}\right) \tilde{x}_{1}(t) \\
& +K_{\tau} C_{1} A_{11}^{t-\tau} \tilde{x}_{1}(t-\tau) \\
& +\left(A_{22}^{0}+K C_{1} A_{12}^{t}\right) \tilde{x}_{2}(t) \\
& +K_{\tau} C_{1} A_{12}^{t-} \tilde{x}_{2}(t-\tau)+B_{20}\left(u+\psi_{0}(t, y, u)\right) \\
& -\left(A_{22}^{0}+K C_{1} A_{12}^{t}\right) K C_{1} \tilde{x}_{1}(t) \\
& -K_{\tau} C_{1} A_{12}^{t-\tau} K_{\tau} C_{1} \tilde{x}_{1}(t-2 \tau)-\left(K C_{1} A_{12}^{t} K_{\tau}\right. \\
& \left.+A_{22}^{0} K_{\tau}+K_{\tau} C_{1} A_{12}^{t-\tau} K\right) C_{1} \tilde{x}_{1}(t-\tau)
\end{aligned}
$$

Note that the last two lines of the previous equation only depend on the output information and thus the following output feedback control law can be defined:

$$
\begin{aligned}
u(t) & =-\left(B_{20}\right)^{-1}\left\{\left(A_{22}^{0}+K C_{1} A_{12}^{0}\right) \tilde{x}_{2}(t)\right. \\
& +K_{\tau} C_{1} A_{12}^{0} \tilde{x}_{2}(t-\tau)+\nu-\left(A_{22}^{0}\right. \\
& \left.+K C_{1} A_{12}^{0}\right) K C_{1} \tilde{x}_{1}(t)-\left(K C_{1} A_{12}^{0} K_{\tau}\right. \\
& \left.+A_{22}^{0} K_{\tau}+K_{\tau} C_{1} A_{12}^{0} K\right) C_{1} \tilde{x}_{1}(t-\tau) \\
& \left.-K_{\tau} C_{1} A_{12}^{0} K_{\tau} C_{1} \tilde{x}_{1}(t-2 \tau)-G_{l} \tilde{x}_{2}(t)\right\}
\end{aligned}
$$

where $G_{l}$ is a Hurwitz matrix. The closed loop system satisfies the following equations:

$$
\begin{aligned}
& \dot{\tilde{x}}_{1}(t)=\left(A_{11}^{t}-A_{12}^{t} K C_{1}\right) \tilde{x}_{1}(t) \\
& \quad-A_{12}^{t} K_{\tau} C_{1} \tilde{x}_{1}(t-\tau)+A_{12}^{t} \tilde{x}_{2}(t) \\
& \dot{\tilde{x}}_{2}(t)=G_{l} \tilde{x}_{2}(t)+\left(A_{21}^{t}+K C_{1} A_{11}^{t}\right) \tilde{x}_{1}(t) \\
& \quad+K_{\tau} C_{1} A_{11}^{t} \tilde{x}_{1}(t-\tau)-\nu+\psi_{1}(t, y, u)
\end{aligned}
$$

where

$$
\begin{aligned}
\psi_{1}(t, y, u)=\sum_{i=1} \lambda_{i}(t)\left[K C_{1} A_{12}^{i} \tilde{x}_{2}(t)\right. \\
\quad+K C_{1} A_{12}^{i} K C_{1} \tilde{x}_{1}(t) \\
\left.\quad-K C_{1} A_{12}^{i} K_{\tau} C_{1} \tilde{x}(t-\tau)\right] \\
\quad+\sum_{i=1} \lambda_{i}(t-\tau)\left[K_{\tau} C_{1} A_{12}^{i} \tilde{x}_{2}(t-\tau)\right. \\
\quad+K_{\tau} C_{1} A_{12}^{i} K C_{1} \tilde{x}_{1}(t-\tau) \\
\left.\quad-K \tau C_{1} A_{12}^{i} K_{\tau} C_{1} \tilde{x}(t-2 \tau)\right] \\
\quad+B_{20} \psi_{0}(t, y, u)
\end{aligned}
$$

Since $\psi_{1}$ depends on $t, y$ and $u$ only, there exist positive functions $\Psi_{2}$ and $\Psi_{21}$ such that:

$$
\left\|\psi_{1}(t, y, u)\right\| \leq\left\|B_{20}\right\| \Psi_{2}(t, y, u)+\Psi_{21}(t, y, u)
$$

The discontinuous control component $\nu$ is still defined by (11) but the gain is now defined by:

$$
\rho(t, y, u)=\left\|B_{20}\right\| \Psi_{2}(t, y, u)+\Psi_{21}(t, y, u)+\delta
$$

where $\delta$ is a positive scalar gain.

Noting that equation (31) is polytopic and of the same form as (31), and that Theorem 2 is linear with respect to the matrix definition, the following result holds:

Theorem 3: System (31) is exponentially stable for given output feedback gains $K$ and $K_{\tau}$ with decay rate $\alpha$ if there exist $P_{1}>0, P_{2}, P_{3}, S_{p}=S_{p}^{T}$, $Q_{p}, R_{p q}=R_{q p}^{T}, p, q=0, \ldots, N$ in $\mathbb{R}^{(n-m) \times(n-m)}$ and $Q_{2}>0 \in \mathbb{R}^{m \times m}$ which satisfy the LMI condition (21) and (18) for all vertices $i=1, . ., M$ with $h=$ $\tau / N$.

Then the following corollary holds:

Corollary 2: An ideal sliding motion takes place in the domain $\Omega$ given by

$$
\begin{gathered}
\left\{\left(\tilde{x}_{1}, \tilde{x}_{2}\right) \in[t-\tau, t] \mapsto R^{n-m} \times R^{m}:\right. \\
\max _{i, j=1, . ., N} \\
\left.\left(\left\|\left(\Lambda_{21}^{i}+K C_{1} \Lambda_{11}^{i}\right)\right\|+\left\|K_{\tau} C_{1} \Lambda_{11}^{j}\right\|\right)\left|\tilde{x}_{1}\right|_{\tau}<\delta-\eta\right\}
\end{gathered}
$$

where $\eta$ is a small scalar satisfying $0<\eta<\delta$.

Proof: The proof is similar to the previous one.

\section{EXAMPLE}

Consider the non-delayed system (3) with the definitions:

$$
\begin{array}{cc}
A_{11}=\left[\begin{array}{ll}
0 & -2 \\
1 & 0.1
\end{array}\right], & A_{12}=\left[\begin{array}{c}
-1 \\
0
\end{array}\right], \\
A_{21}=\left[\begin{array}{cc}
-0.1 & -1
\end{array}\right], & A_{22}=\left[\begin{array}{l}
1 \\
0
\end{array}\right], \\
C=\left[\begin{array}{ll}
0 & 0 \\
1 & 0 \\
0 & 1
\end{array}\right]^{T}, & B=\left[\begin{array}{l}
0 \\
0 \\
1
\end{array}\right] .
\end{array}
$$

As in [2], this system is not output stabilizable using traditional static (ie. non delayed output feedback). The objective remains here to design the controller (10) with appropriate gains $K, K_{\tau} \in \mathbb{R}$ and an artificial delay $\tau$ such that the closed-loop system is exponentially stable with decay rate $\alpha$.

\section{A. Design of the output feedback}

This section proposes a method to obtain the optimal controller $\left(K, K_{\tau}, \tau\right)$. The idea is to test if, for a set of values of $K$ and $K_{\tau}$, the LMIs from Theorem 2 have a solution and if it is possible to find the delay which ensures the greatest exponential decay rate.

After checking the resolution of the LMIs from Theorem 2, a solution can only be found when $K$ 
lies in the interval $[-6 ; 2]$ and $K_{\tau}$ in $[0 ; 8]$. For each value of the gains $K$ and $K_{\tau}$, an optimization process, detailed in Appendix $B$, is used to obtain the best value of $\alpha$ by tuning $\tau$ upwards from zero until the LMIs are not satisfied. The optimal delay will be the one which delivers the largest $\alpha$, using the same method as in Example 1. For this particular example the optimization problem is reduced to the following one:

$$
\left.\begin{array}{c}
\alpha_{\max }= \\
\left(K, K_{\tau}\right) \in[-6 ; 2] \times[0 ; 8] \\
\text { such that (18) and (21) are satisfied }
\end{array}\right\}
$$

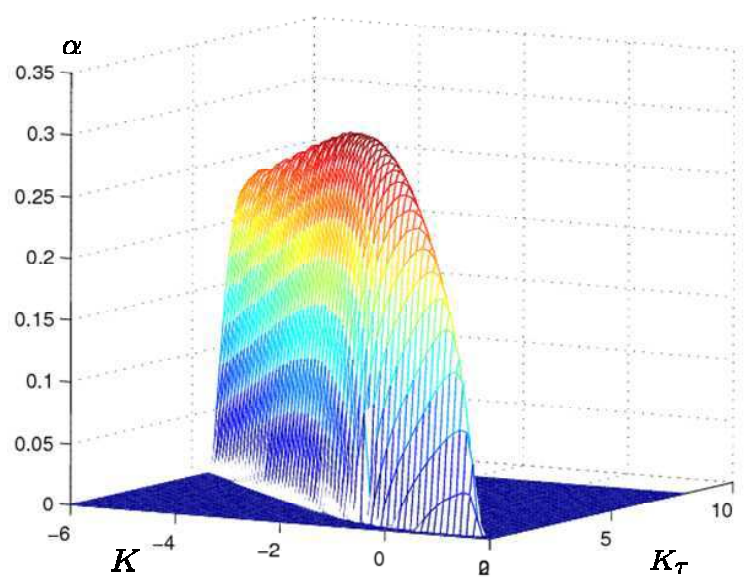

Fig. 2. Maximum decay rate $\alpha$ with respect to $K$ and $K_{\tau}$ for $N=1$

Figure 2 shows the relation between the output feedback gains and the decay rate $\alpha$ using Theorem 2 with $N=1$. The size of the set increases when the discretization number $N$ increases. Figure 2 also shows that the graph has a maximum at $K=-1.625$ and $K_{\tau}=2.625$. This selection of gains $K$ and $K_{\tau}$ ensures the system is exponentially stable with a decay rate $\alpha=0.3$. The corresponding optimal delay is $\tau=0.3$.

For $N=3$ the optimized gains are $K=-2.23$ and $K_{\tau}=3.06$. The corresponding optimal delay is $\tau=0.43$. For these parameters the decay rate is $\alpha=0.612$. Theorem 2 also ensures for $N=6$ that the same gains $K=-2.23$ and $K_{\tau}=3.06$ exponentially stabilize the system (3) with a decay rate $\alpha=0.826$ with the optimal delay $\tau=0.45$.

Remark 8: For $N=3$, the computation of the conditions from Theorem 2 become very heavy. The optimization problem has not been tested for $N \geq 3$.

\section{B. Simulation results}

In the results which follow system (3) is controlled using (10) with $K=-2.23, K_{\tau}=3.06$ and $\tau=0.45$.
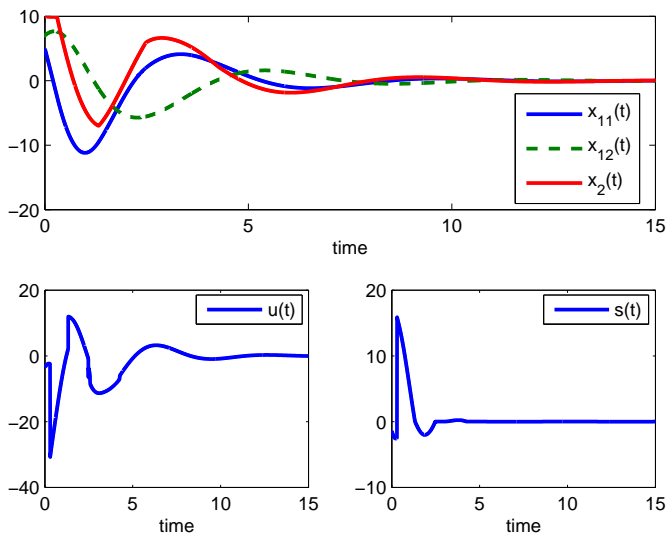

Fig. 3. Simulation results for $K=-2.23, K_{\tau}=3.06$ and $\tau=0.45$

Figure 3 shows the state, the input and the sliding function. The state converges exponentially to $x(t)=0$ with an exponential decay rate $\alpha=0.826$. The sliding function converges to $\tilde{x}_{2}=0$ in finite time. The evolution of the control signal is shown in Figure 3.
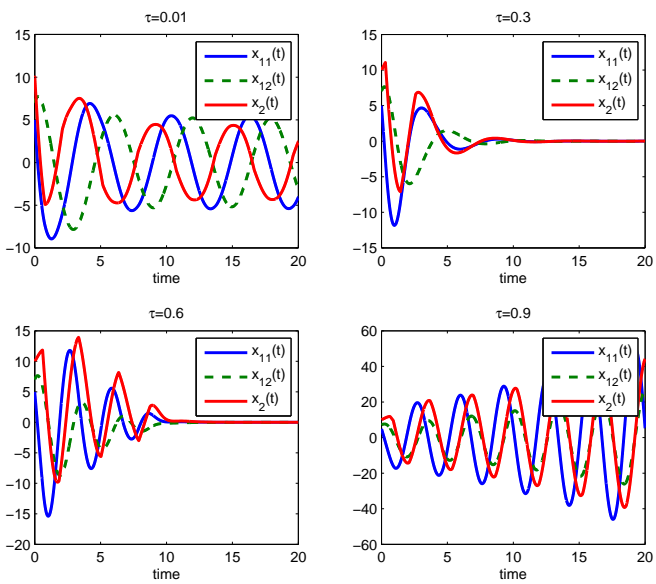

Fig. 4. Simulation results for different values of the delay $\tau$

In Figure 4, different delays are used to show robustness to changes in the delay. For too small values, e.g. $\tau=0.01$, or too large a delay e.g. $\tau=0.9$, the system is unstable. However when $\tau=0.3$ or 0.6 , which are sufficiently close to the optimal delay $\tau=0.45$, the system is still stable. This behavior is consistent with the results of Example 1 (see Figure 
1). For given $K$ and $K_{\tau}$, exponential stability is ensured for delays sufficiently close to the optimal value of the delay, but the exponential decay rate is lower.

\section{CONCLUSION}

A new sliding mode controller has been suggested for systems for which finding a traditional static output feedback sliding mode controller is not possible. The controller introduces a stabilizing delay in the closed loop system. The controller is simple and does not require heavy real-time computation. An example is used to demonstrate a method to design the gains and the delay of the controller. The robustness with respect to the delay has been shown in the example. A straightforward extension ensures robust stabilization with respect to disturbances and to parameter uncertainties.

\section{REFERENCES}

[1] S. Bag, S.K. Spurgeon, and C. Edwards, Output feedback sliding mode design for linear uncertain systems, Proceedings of the IEE, Part D, 144, 1997, pp. 209-216.

[2] C. Edwards and S.K. Spurgeon, Sliding mode stabilization of uncertain systems using only output information, Int. J. of Contr. 62 (1995), no. 5, 1129-1144.

[3] _ Compensator based output feedback sliding mode control design, International Journal of Control 71 (1998), 601614.

[4] Sliding mode control: Theory and applications, Taylor \& Francis, 1998.

[5] C. Edwards, S.K. Spurgeon, and R.G. Hebden, On the design of sliding mode output feedback controllers, Int. Journal of Control 76 (2003), no. 9-10, 893-905.

[6] E. Fridman, Stability of linear descriptor systems with delay: A Lyapunov-based approach, Journal of Mathematical Analysis and Applications 273 (2002), no. 1, 24-44.

[7] - Descriptor discretized Lyapunov functional method : Analysis and design, IEEE Trans. on Automatic Control 51(5) (2006), 890 - 897.

[8] _ Stability of systems with uncertain delays: a new "complete" Lyapunov-Krasovskii functional, IEEE Trans. on Automatic Control 51(5) (2006), 885 - 890.

[9] E. Fridman and U. Shaked, A descriptor system approach to $H^{\infty}$ control of linear time-delay systems, IEEE Trans. on Automatic Control 47 (2002), no. 2, 253-270.

[10] K. Gu, Discretized LMI set in the stability problem of linear uncertain time-delay systems, Int. J. of Control 68 (1997), 923 $-934$.

[11] _ A further refiniment of discretized Lyapunov functional method for the stability of time-delay systems, Int. J. of Control 74 (2001), 967 - 976.

[12] K. Gu, V.-L. Kharitonov, and J. Chen, Stability of time-delay systems, Birkhauser, 2003.

[13] E.W. Jacobsen and G. Cedersund, Structural robustness of biochemical network models-with application to the oscillatory metabolism of activated neutrophils, IET Systems Biology 2 (2008), 39-47.
[14] V.L. Kharitonov and S. Mondié, Exponential estimates for neutral time-delay systems: an LMI approach, IEEE Transactions on Automatic Control 50 (2005), no. 5, 666-670.

[15] W. Michiels, S.-I. Niculescu, and L. Moreau, Using delays and time-varying gains to improve the static output feedback stabilizability of linear systems : a comparison, IMA Journal of Mathematical Control and Information 21 (2004), no. 4, 393418.

[16] S. Mondié and V.L. Kharitonov, Exponential estimates for retarded time-delay systems: an LMI approach, IEEE Trans. on Automatic Control 50 (2005), no. 2, 268-273.

[17] S.-I. Niculescu and C. T. Abdallah, Delay effects on static output feedback stabilization, Proceedings of the $39^{\text {th }}$ IEEE Conference on Decision and Control (Sydney, Autralia), December 2000 .

[18] S.-I. Niculescu, K. Gu, and C. T. Abdallah, Some remarks on the delay stabilizing effect in SISO systems, Proceedings of the American Control Conference (Denver, USA), June 2003.

[19] A. Seuret, M. Dambrine, and J.-P. Richard, Robust exponential stabilization for systems with time-varying delays, 5th Workshop on Time Delay Systems, September 2004.

[20] A. Seuret, E. Fridman, and J.-P. Richard, Sampled-data exponential stabilization of neutral systems with input and state delays, IEEE MED 2005, $13^{\text {th }}$ Mediterranean Conference on Control and Automation, June 2005.

[21] S. Xu, J. Lam, and M. Zhong, New exponential estimates for time-delay systems, IEEE Transactions on Automatic Control 51 (2006), no. 9, 1501-1505.

\section{APPENDIX}

\section{A. Proof of Theorem 1}

The following is not a new result, but the inclusion of a sketch of the proof of the discretization theorem is included to improve readability. Based on the results of [8], the first part of the proof of exponential stability consists of expressing the derivative of the Lyapunov Krasovskii functional appropriately. The next step of the proof focusses on the application of the discretization process of $\mathrm{Gu}[10]$.

Consider system (16) in a descriptor representation with the extended state vector $\bar{x}_{\alpha}(t)=$ $\operatorname{col}\left\{x_{\alpha}(t), \dot{x}_{\alpha}(t)\right\}$. This can be written as:

$$
\begin{aligned}
{\left[\begin{array}{cc}
I_{n} & 0_{n} \\
0_{n} & 0_{n}
\end{array}\right] \dot{\bar{x}}_{\alpha}(t) } & =\left[\begin{array}{cc}
0_{n} & I_{n} \\
A_{0}+\alpha I_{n} & -I_{n}
\end{array}\right] \bar{x}_{\alpha}(t) \\
& +\left[\begin{array}{c}
0_{n} \\
e^{\alpha \tau} A_{1}
\end{array}\right] x_{\alpha}(t-\tau)
\end{aligned}
$$

The first term of the Lyapunov Krasovskii functional $V_{1 \alpha}$ can be rewritten in the form:

$$
x_{\alpha}^{T}(t) P_{1} x_{\alpha}(t)=\bar{x}_{\alpha}(t)\left[\begin{array}{cc}
I_{n} & 0_{n} \\
0_{n} & 0_{n}
\end{array}\right] P \bar{x}_{\alpha}(t)
$$

where $P=\left[\begin{array}{ll}P_{1} & 0_{n} \\ P_{2} & P_{3}\end{array}\right]$ 
Differentiating the Lyapunov functional $V_{1 \alpha}$ along the trajectories of (16) leads to:

$$
\begin{aligned}
\dot{V}_{1 \alpha}(t) & =2 \dot{x}_{\alpha}^{T}(t)\left[P_{1} x_{\alpha}(t)+\int_{-\tau}^{0} Q(\zeta) x_{\alpha}(t+\zeta) d \zeta\right] \\
& +2 x_{\alpha}^{T}(t) \int_{-\tau}^{0} Q(\zeta) \dot{x}_{\alpha}(t+\zeta) d \zeta \\
& +2 \int_{-\tau}^{0} \int_{-\tau}^{0} \dot{x}_{\alpha}^{T}(t+s) R(s, \zeta) d s x_{\alpha}(t+\zeta) d \zeta \\
& +2 \int_{-\tau}^{0} \dot{x}_{\alpha}^{T}(t+\zeta) S(\zeta) x_{\alpha}(t+\zeta) d \zeta
\end{aligned}
$$

Rewriting the first term of (33) using the descriptor representation [6], and integrating by parts in (33), the following equality can be established:

$$
\begin{aligned}
\dot{V}_{1 \alpha}(t) & =\xi^{T}(t) \Xi_{\alpha} \xi(t)+2 \dot{x}_{\alpha}^{T}(t) \int_{-\tau}^{0} Q(\zeta) x_{\alpha}(t+\zeta) d \zeta \\
- & \int_{-\tau}^{0} x_{\alpha}^{T}(t+\zeta) \dot{S}(\zeta) x_{\alpha}(t+\zeta) d \zeta \\
- & \int_{-\tau}^{0} \int_{-\tau}^{0} x_{\alpha}^{T}(t+s)\left(\frac{\partial}{\partial s} R(s, \zeta)+\frac{\partial}{\partial \zeta} R(s, \zeta)\right) \\
& x_{\alpha}(t+\zeta) d s d \zeta \\
+ & 2 x_{\alpha}^{T}(t) \int_{-\tau}^{0}[-\dot{Q}(\zeta)+R(0, \zeta)] x_{\alpha}(t+\zeta) d \zeta \\
- & 2 x_{\alpha}^{T}(t-\tau) \int_{-\tau}^{0} R(-\tau, \zeta) x_{\alpha}(t+\zeta) d \zeta
\end{aligned}
$$

where $\xi(t)=\operatorname{col}\left\{\bar{x}_{\alpha}(t), x_{\alpha}(t-\tau)\right\}$ and $\Xi_{\alpha}$ has the form in (19) with $Q(0), Q(-\tau), S(0)$ and $S(-\tau)$ instead of $Q_{0}, Q_{N}, S_{0}$ and $S_{N}$ respectively. The Lyapunov functional is now expressed in an appropriate representation to apply the discretization.

The discretization divides the delay interval $[-\tau, 0]$ into $N$ segments $\left[\theta_{p}, \theta_{p-1}\right], p=1, . ., N$ of equal length $h=\tau / N$. This divides the square

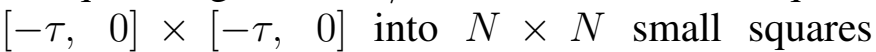
$\left[\theta_{p}, \theta_{p-1}\right] \times\left[\theta_{p}, \theta_{p-1}\right]$. Each small square is further divided into two triangles.

The continuous matrix functions $Q(\xi)$ and $S(\xi)$ are chosen to be linear within each interval and the continuous matrix functions $R(s, \xi)$ is chosen to be linear within each triangle. The proposed matrix functions are:

$$
\begin{aligned}
& Q\left(\theta_{p}+\beta h\right)=(1-\beta) Q_{p}+\beta Q_{p-1}, \\
& S\left(\theta_{p}+\beta h\right)=(1-\beta) S_{p}+\beta S_{p-1} \\
& R\left(\theta_{p}+\beta h, \theta_{q}+\gamma h\right)= \\
& \begin{cases}(1-\beta) R_{p q}+\gamma R_{(p-1)(q-1)}+(\beta-\gamma) R_{(p-1) q}, & \beta \geq \gamma \\
(1-\gamma) R_{p q}+\beta R_{(p-1)(q-1)}+(\gamma-\beta) R_{(p-1) q}, & \beta \leq \gamma\end{cases}
\end{aligned}
$$

for $0 \leq \beta \leq 1$ and $0 \leq \beta \leq 1$. Simple definitions of the derivative of the matrix functions can be obtained which are, for appropriate $p$ and $q$ :

$$
\begin{gathered}
\dot{S}(\xi)=1 / h\left(S_{p-1}-S_{p}\right), \\
\dot{Q}(\xi)=1 / h\left(Q_{p-1}-Q_{p}\right), \\
\frac{\partial}{\partial s} R(s, \xi)+\frac{\partial}{\partial \xi} R(s, \xi)=1 / h\left(R_{(p-1)(q-1)}-R_{p q}\right)
\end{gathered}
$$

Thus, the Lyapunov Krasovskii functional is completely determined by the matrices $P_{1}, S_{p}, Q_{p}$ and $R_{p q}, p, q=0, . ., N$. From [12], the condition $V_{1 \alpha} \geq \epsilon\left\|x_{\alpha}\right\|$ is satisfied if LMI (18) is satisfied. Using conditions (35), the following equations hold:

$$
\begin{aligned}
& 2 \dot{x}_{\alpha}(t) \int_{-\tau}^{0} Q(\xi) x(t+\xi) d \xi \\
& =2 \dot{x}_{\alpha}(t) \sum_{p=1}^{N} \int_{0}^{1}\left[(1-\beta) Q_{p}+\beta Q_{p-1}\right] x_{\alpha}\left(t_{p}^{\beta}\right) d \beta \\
& =2 \dot{x}_{\alpha}(t) \sum_{p=1}^{N} \int_{0}^{1}\left[( 1 - \beta ) \left(Q_{p}^{s}\right.\right. \\
& \left.\left.\quad+Q_{p}^{a}\right)+\beta\left(Q_{p}^{s}-Q_{p}^{a}\right)\right] x_{\alpha}\left(t+\theta_{p}+\beta h\right) d \beta
\end{aligned}
$$

where $t_{p}^{\beta}=t+\theta_{p}+\beta h, Q_{p}^{s}=\left(Q_{p}+Q_{p-1}\right) / 2$ and $Q_{p}^{a}=\left(Q_{p}-Q_{p-1}\right) / 2$. Then equations (19), (34) and (35) imply [12]:

$$
\begin{aligned}
\dot{V}_{1 \alpha}(t)= & \zeta^{T}(t) \Xi_{\alpha} \zeta(t)-\int_{0}^{1} \phi^{T}(\beta) \dot{S}_{d} \phi(\beta) d \beta \\
& -\int_{0}^{1} \int_{0}^{1} \phi(\beta) R_{d} \phi(\gamma) d \beta d \gamma \\
& +2 \zeta(t) \int_{0}^{1}\left[D^{s}+(1-2 \alpha) D^{a}\right] \phi(\beta) d \beta
\end{aligned}
$$

where $\phi(\beta)=\operatorname{col}\{x(t-h+\beta h), x(t-2 h+$ $\beta h), . ., x(t-N h+\beta h)\}$. Applying Proposition 5.21 from [12], it can be concluded that $\dot{V}_{1 \alpha}(t)<0$ if LMI (17) is satisfied.

\section{B. Optimization programs}

The following table presents a schematic of the optimization program developed for Theorem 1 and 2. The variables $\epsilon_{\tau}$ and $\epsilon_{K}$ represent the grid size used during the search.

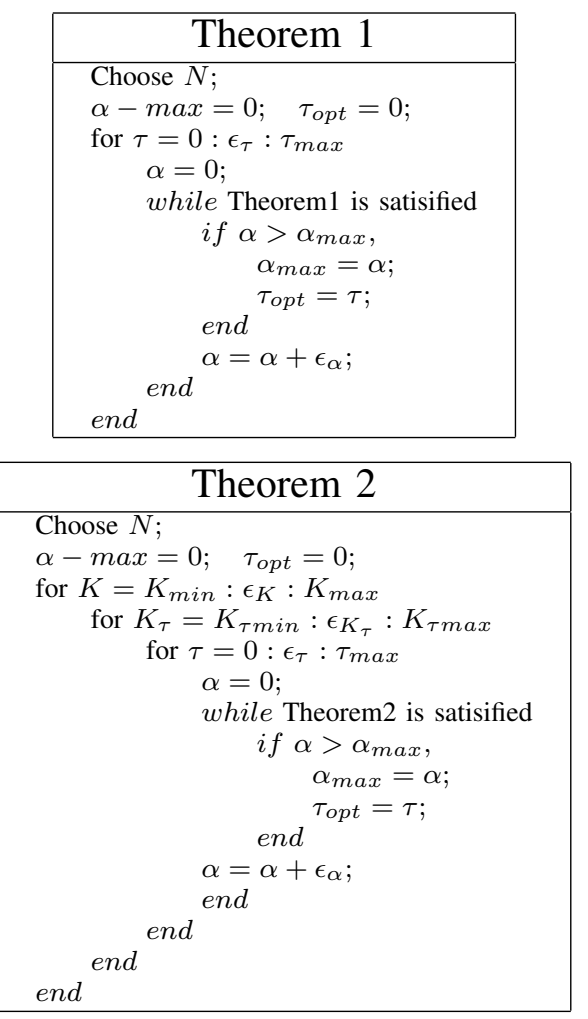

